Orthopäde 2009 · 38:237

DOI 10.1007/s00132-009-1417-7

(c) Springer Medizin Verlag 2009

\author{
V. Ewerbeck \\ Stiftung Orthopädische Universitätsklinik, Abteilung Orthopädie I, \\ Orthopädische Universitätsklinik Heidelberg
}

\title{
Wissenschaft und Praxis - eine gute Mischung
}

be unserer Zeitschrift ist ausschließlich Originalen und Kasuistiken gewidmet, die nichts mit Grundlagenforschung zu tun haben. Sie bearbeiten Fragestellungen aus dem Kerngeschäft unseres klinischen Alltags. Die Frage, wie viel Modularität in der Revisionsendoprothetik der Hüfte sinnvoll, erlaubt und erfolgreich ist, ist hoch aktuell. Sie kann durch die hier publizierte Arbeit nicht abschließend beantwortet werden, aber es werden belastbare Zahlen geliefert, aus denen sich eine eigene Meinung machen lässt. Ähnliches gilt auch für die Fragestellung nach der bestmöglichen operativen Therapie der bakteriellen Spondylodiszitis, der Behandlung juveniler Knochenzysten oder der Frage, welchen Nutzen die diagnostische Arthroskopie vor kniegelenksnahen Korrekturosteotomien bei unikompartimenteller Gonarthrose hat. Für alle diese Fragen gibt es in diesem Heft wichtige Hinweise.

Ich bin ein überzeugter Anhänger von gut aufbereiteten Kasuistiken. Ich lerne am meisten durch den entweder selbst erlebten, oder gut dokumentierten klinischen Fall und zwar unabhängig davon, ob das Behandlungsergebnis gut oder schlecht war. Je eindringlicher das Erlebnis oder die Präsentation, desto eher besteht die Chance, dass das Lernziel erreicht und in meinem Langzeitgedächtnis verfügbar gehalten wird. In diesem Sinne darf ich Ihnen, liebe Leser auch die Lektüre der drei publizierten Fallbeschreibungen ans Herz legen.

Vielleicht wird ja auch die eine oder andere Arbeitsgruppe durch dieses Heft stimuliert, eigene klinische Fragestellungen in Angriff zu nehmen, ein gutes Manuskript zu erstellen und es bei der Schriftleitung einzureichen. Unser Impactfaktor steigt, und die deutsche Sprache ist immer noch eine schöne Sprache. Sie liest sich einfach gut und erreicht eine Menge Leser.

In diesem Sinne verbleibe ich mit herzlichen kollegialen Grüßen

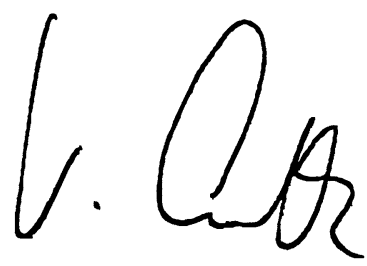

V. Ewerbeck

Korrespondierender Autor:

Prof. Dr. med. V. Ewerbeck

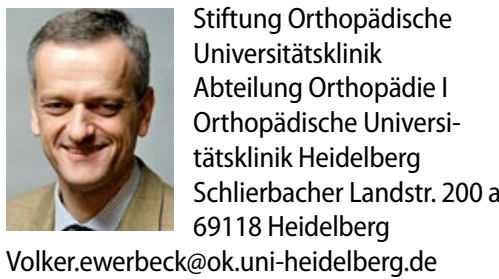

Volker.ewerbeck@ok.uni-heidelberg.de 